\title{
Adaptive discrepancy based control of continuous fluidized bed spray granulation with internal classification
}

\author{
Stefan Palis ${ }^{1, *}$, Andreas Bück ${ }^{1}$, Achim Kienle ${ }^{1,2}$ \\ 1 Otto-von-Guericke-Universität, \\ Universitätsplatz 2, D-39106 Magdeburg, Germany \\ 2 Max-Planck-Institut für Dynamik komplexer technischer Systeme \\ Sandtorstrasse 1, D-39106 Magdeburg, Germany
}

\begin{abstract}
This paper is concerned with adaptive stabilization of open loop unstable fluidized bed spray granulation with internal product classification by means of nonlinear feedback control. Since the process model is represented by a nonlinear partial integro-differential equation, direct stabilization of the particle size distribution in a $L_{p}$ or $L_{\infty}$ norm is difficult. To overcome this problem a stability notion using two generalized distance measures, the discrepancies, is used. It is shown that the adaptive version of the resulting discrepancy based control law is able to cope with uncertainties present in industrial applications.
\end{abstract}

\section{INTRODUCTION}

Fluidized bed spray granulation is a particulate process, where a bed of particles is fluidized, while simultaneously injecting a solid matter solution. Due to high process air temperature, the fluid evaporates and the remaining solid material either contributes to growth of already existing particles or forms new nuclei. As product particles should have a certain size an additional product classification is required. This can be either done by external classification using sieves with corresponding recycle of the over- and undersized fraction [2] or by internal classification using an air sifter with countercurrent flow as depicted in Fig. 1 , which will be the topic of this contribution. A corre-

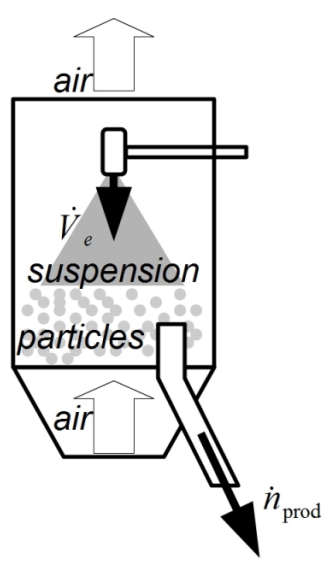

Fig. 1. Process scheme

sponding process model has been proposed by Vreman et. al. [4]. In particular, it has been shown that for certain ranges of the operating parameters regions of instability exist, resulting in nonlinear oscillations of the particle size distribution. These oscillations give undesired time behavior of product quality. Similar patterns of behavior have been observed for other particulate processes as continuous fluidized bed spray granulation with external product classification and material recycles (e.g. [3]) and crystallization processes (e.g. [13]). So far, main emphasis was on crystallization processes. Here several approaches for stabilizing control design have been proposed, using linear (e.g. [10]) and nonlinear (e.g. [11]) lumped models or linear (e.g. [12]) and nonlinear infinite dimensional models as in [6]. For fluidized bed spray granulation with external product classification linear and nonlinear control design procedures have been proposed in $[5,7]$.

In this contribution control design based on the nonlinear infinite dimensional model of the fluidized bed spray granulation with internal product classification is investigated using a generalized stability theory in the sense of two generalized distances, the discrepancies. In order to account for strong variations present in the industrial application the discrepancy based control approach will be extended by an adaptation mechanism.

\section{CONTINUOUS FLUIDIZED BED SPRAY GRANULATION WITH CLASSIFYING PRODUCT WITHDRAWAL}

A continuous fluidized bed spray granulator with internal product classification as depicted in Fig. 1 consists of a granulation chamber, where the particle population is fluidized through an air stream and coated by injecting a suspension $\dot{V}_{e}$. The particle growth associated to the layering process has been described in [1]. In Vreman et. al. [4] the growth rate has been slightly modified to account for internal nucleation. In this extended approach only a certain part of the injected suspension $\left((1-b) \dot{V}_{e}\right)$ contributes to the particle growth

$$
G=\frac{2(1-b) \dot{V}_{e}}{\pi \int_{0}^{\infty} L^{2} n d L}=\frac{2(1-b) \dot{V}_{e}}{\pi \mu_{2}}
$$


with $b \in[0,1]$. The rest of the suspension $\left(b \dot{V}_{e}\right)$ results in new nuclei due to drying spray droplets, which completely dry before hitting existing particles in the bed. Here, it is assumed that the size distribution of the formed nuclei is a normal distribution with a mean diameter $L_{0}$ as depicted in Fig. 2.

$$
B=\frac{b \dot{V}_{e}}{\frac{1}{6} \pi} \frac{e^{-\frac{\left(L-L_{0}\right)^{2}}{a^{2}}}}{\int_{0}^{\infty} L^{3} e^{-\frac{\left(L-L_{0}\right)^{2}}{a^{2}}} d L}=\frac{b \dot{V}_{e}}{\frac{1}{6} \pi} n_{B}(L)
$$

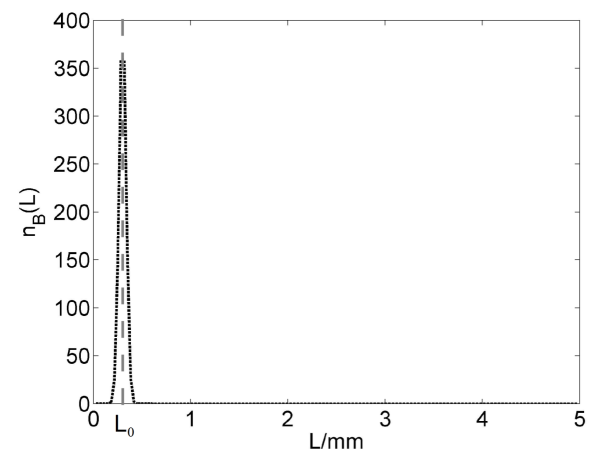

Fig. 2. Normal distribution of formed nuclei $n_{B}(L)$

It is assumed that the nucleation parameter $b$ determining how much of the injected suspension results in new particles depends only on the bed height $h$, which can be obtained from

$$
h=\frac{V}{(1-\varepsilon) A},
$$

where $\varepsilon$ is the bed porosity. In the following the bed porosity $\varepsilon$ is assumed to be constant. The free distance for the spray droplets decreases with increasing bed height $h$ resulting in a decreasing nuclei formation. For a bed higher or equal to the nozzle height a constant minimal nucleation parameter $b=b_{\infty}$ is assumed. The maximum of the nucleation parameter $b=1$ is reached for a minimum bed height of 0 resulting in a pure spray drying process, i.e. $100 \%$ of the injected suspension forms new particles. For bed heights between the two extreme situations $h=0$ and $h=h_{n o z}$ the nucleation parameter $b$ is interpolated linearly [4].

$$
b=b_{\infty}+\max \left(0,\left(1-b_{\infty}\right) \frac{h_{n o z}-h}{h_{n o z}}\right)
$$

In order to guarantee a continuous process operation particles are continuously removed through an air sifter with countercurrent flow. Due to the particle size specific sinking velocity large particles pass the air sifter while small particles are reblown into the granulation chamber. The associated non-ideal separation function $T$ shown in Fig. 3

$$
T(L)=\frac{\int_{0}^{L} e^{-\frac{\left(L^{\prime}-L_{1}\right)^{2}}{a^{2}}} d L^{\prime}}{\int_{0}^{\infty} e^{-\frac{\left(L-L_{1}\right)^{2}}{a^{2}}} d L}
$$

results with the drain $K$ in the following outlet flow

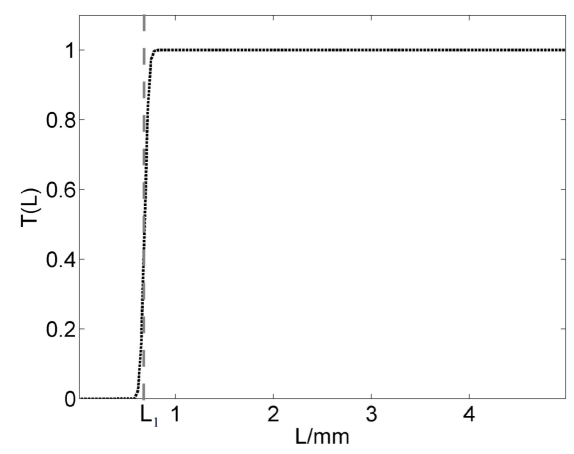

Fig. 3. Non-ideal separation function $T$ due to classifying product removal

$$
\dot{n}_{\text {prod }}=K T(L) n \text {. }
$$

To describe the process, a population balance model for the particle size distribution has been proposed in [4] consisting of the following particle fluxes

- $B$ particle flux due to nuclei formation,

- $\dot{n}_{\text {prod }}$ particle flux due to product removal,

and size independent particle growth associated with the particle growth rate $G$.

$$
\frac{\partial n}{\partial t}=-G \frac{\partial n}{\partial L}-\dot{n}_{\text {prod }}+B
$$

For numerical simulation the model equations are semidiscretized with the finite volume method (1st order upwind flux discretization) with 150 grid points. The model parameters used are given in Table 1.

\begin{tabular}{ll}
\hline$A$ & $5 \cdot 10^{6} \mathrm{~mm}^{2}$ \\
$h_{n o z}$ & $440 \mathrm{~mm}$ \\
$\varepsilon$ & 0.5 \\
$\dot{V}_{e}$ & $1.67 \cdot 10^{5} \frac{\mathrm{mm}^{3}}{\mathrm{~s}}$ \\
$b_{\infty}$ & 0.028 \\
$L_{0}$ & $0.3 \mathrm{~mm}$ \\
$L_{1}$ & $0.7 \mathrm{~mm}$ \\
$K$ & $1.92 \cdot 10^{-4} \frac{1}{\mathrm{~s}}$ \\
\hline \multicolumn{2}{c}{ Table 1. Plant parameters }
\end{tabular}

Interesting dynamical behavior can be observed, when starting with an initial particle size distribution equal to the steady state particle size distribution for $\dot{V}_{e}=$ $16700 \frac{\mathrm{mm}^{3}}{\mathrm{~s}}$. For sufficiently high suspension injection rates $\left(\dot{V}_{e}=0.96 \cdot \dot{V}_{e, 0}\right)$ and an associated bed height higher than the nozzle height, transition processes decay and the particle size distribution reaches a stable steady state as shown in Fig. 4 (left). Decreasing the suspension injection rate $\left(\dot{V}_{e}=0.9 \cdot \dot{V}_{e, 0}\right)$ the steady state becomes unstable giving rise to nonlinear oscillations as depicted in Fig. 4 (right). Here, the associated mechanism is as follows:

- For a bed height smaller than the nozzle height spray drying causes an increased nuclei production

- resulting in an increasing number of smaller particles and a reducing growth rate.

- When the bed height reaches the nozzle height the production of nuclei becomes small and remains constant, resulting in a higher growth rate. 
- However, when the peak of the particle size distribution reaches the critical sifter radius $L_{1}$ the associated particles are removed from the granulator resulting in a decrease of the bed height below the nozzle height and hence the process repeats.

In contrast, a high suspension rate gives a constantly high production of nuclei and a high growth rate. Therefore, the bed height remains bigger than the nozzle height and after a transition time the steady state particle size distribution is reached and no oscillations occur.
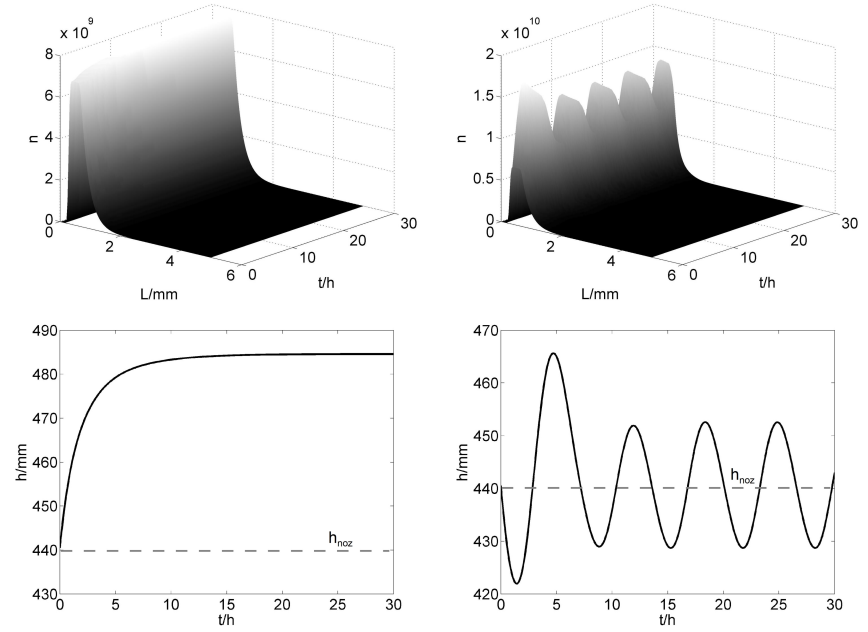

Fig. 4. Open loop simulations in the stable (left) and unstable (right) region

A detailed one-parameter bifurcation analysis (Fig. 5) confirms the previously described process time behavior and its dependence on the chosen suspension injection rate $\dot{V}_{e}$. For sufficiently high values of $\dot{V}_{e}$ the particle size distribution reaches a stable steady state. Decreasing the suspension injection rate $\dot{V}_{e}$ to a critical value and below leads to a loss of stability for the steady state solution and a stable limit cycle occurs. The stable limit cycle is associated to undesired self-sustained oscillations.

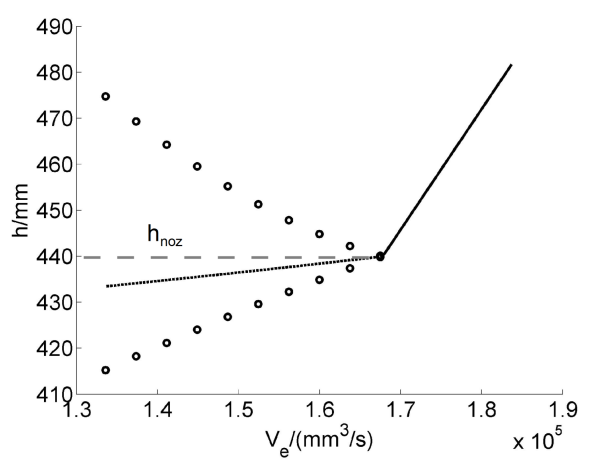

Fig. 5. One parameter bifurcation diagrams depending on $\dot{V}_{e}$

So far, it has been assumed that the suspension injection rate $\dot{V}_{e}$ is equal to the effective injection rate of solid material, which contributes either to particle growth or to nuclei formation. In an industrial setting this is however not the case as here the effective suspension injection rate $\dot{V}_{e}$ is coupled to the specific drying conditions and may vary due to variations in the liquid phase, e.g. varying mass fraction. Hence, in the following the effective suspension injection rate $\dot{V}_{e}$ will be replaced by the suspension injection rate $\dot{V}_{s}$ and an unknown parameter $k$, which accounts for the mentioned variations and uncertainties.

$$
\dot{V}_{e}=k \dot{V}_{s}
$$

In order to stabilize the fluidized bed spray granulation with internal product classification in the presence of the mentioned uncertainties the control approach presented in [9] will be extended by an adaptation mechanism guaranteeing closed loop stability. As we showed by a controllability analysis [8] the suspension injection rate $\dot{V}_{s}$ can be used as a control input. This is in accordance to our earlier results on control of fluidized bed spray granulation with external classification and mill cycle [5, 7], where we choose the mill grade as the principal bifurcation parameter as control input.

\section{STABILITY WITH RESPECT TO TWO DISCREPANCIES}

Over the last decades different methods for the stabilization of systems with distributed parameters have been developed. Most of them are based on the solution of the system itself or at least the desired error system, i.e. the system in closed loop operation. In the backstepping approach (e.g. [14]) for example the control input is designed such that it maps the original system onto a desired stable error system. Whereas in the works of Bastin et. al. (e.g. $[15,16])$ stability is proven using the solution derived with the method of characteristics.

Here, the population balance is a nonlinear partial integrodifferential equation with limited control input and a lack of solution theory. Hence, in this case a transformation to a desired error system with known classical stability behavior is hardly possible. As has been shown in previous contributions $[5,6,9]$ this problem is however solvable by introducing a generalized stability notion, i.e. stability with respect to two distance measures, the discrepancies. In the following the most important properties and facts on stability with respect to two discrepancies are stated in accordance to $[19,20,21]$. Here, the process $\varphi(., t)$ is a solution of the distributed parameter system and $\varphi_{0}=0$ an equilibrium of the system.

\section{Definition 1. Discrepancy}

A discrepancy is a real valued functional $\rho=\rho[\varphi(., t), t]$ with the following properties

(1) $\rho(\varphi, t) \geqslant 0$

(2) $\rho(0, t)=0$

(3) for an arbitrary process $\varphi=\varphi(., t)$ the discrepancy $\rho(\varphi(., t), t)$ is continuous with respect to $t$.

(4) introducing a second discrepancy $\rho_{0}(\varphi)$ with $\rho_{0}(\varphi) \geqslant$ 0 and $\rho_{0}(0)=0$. Than the discrepancy $\rho(\varphi(., t), t)$ is continuous at time $t=t_{0}$ with respect to $\rho_{0}$ at $\rho_{0}=0$, if for every $\varepsilon>0$ and $t_{0}>0$ there exists a $\delta\left(\varepsilon, t_{0}\right)>0$, such that from $\rho_{0} \leqslant \delta\left(\varepsilon, t_{0}\right)$ follows $\rho<\varepsilon$.

According to this definition a discrepancy has not all properties of a metric, e.g. symmetry $d(x, y)=d(y, x)$ or triangular inequality $d(x, y) \leq d(x, z)+d(z, y)$. In addition, it has not to satisfy the important property of 
definiteness, i.e. a vanishing discrepancy $\rho(\varphi, t)=0$ does not automatically imply $\varphi=0$.

Definition 2. Stability with respect to two discrepancies $\rho$ and $\rho_{0}$

The equilibrium $\varphi_{0}=0$ is stable in the sense of Lyapunov with respect to the two discrepancies $\rho$ and $\rho_{0}$ for all $t \geq t_{0}$ if for every $\varepsilon>0$ and $t_{0} \geq 0$ there exists a $\delta=\delta\left(\varepsilon, t_{0}\right)>0$, such that for every process $\varphi(., t)$ with $\rho_{0}<\delta\left(\varepsilon, t_{0}\right)$ follows $\rho<\varepsilon$ for all $t \geq t_{0}$. If in addition $\lim _{t \rightarrow \infty} \rho=0$, than the equilibrium $\varphi_{0}$ is called asymptotically stable in the sense of Lyapunov with respect to the two discrepancies $\rho$ and $\rho_{0}$.

In order to establish a relationship between stability with respect to two discrepancies and the existence of a Lyapunov functional $V$ the notions of positivity and positive definiteness of a functional with respect to a discrepancy have been introduced.

Definition 3. Positivity with respect to a discrepancy $\rho$

The functional $V=V[\varphi, t]$ is called positive with respect to the discrepancy $\rho$, if $V \geq 0$ and $V[0, t]=0$ for all $\varphi$ with $\rho(\varphi, t)<\infty$.

Definition 4. Positive definiteness with respect to a discrepancy $\rho$

The functional $V=V[\varphi, t]$ is positive definite with respect to a discrepancy $\rho$, if $V \geqslant 0$ and $V[0, t]=0$ for all $\varphi$ with $\rho(\varphi, t)<\infty$ und for every $\varepsilon>0$ there exists a $\delta=\delta(\varepsilon)>0$, such that $V \geq \delta(\varepsilon)$ for all $\varphi$ with $\rho[\varphi, t] \geq \varepsilon$.

The following two theorems state the conditions for a function $V$ guaranteeing (asymptotical) stability with respect to two discrepancies.

Theorem 5. [20] The process $\varphi$ with the equilibrium $\varphi_{0}=$ 0 is stable with respect to the two discrepancies $\rho$ and $\rho_{0}$ if and only if there exists a functional $V=V[\varphi, t]$ positive definite with respect to the discrepancy $\rho$, continuous at time $t=t_{0}$ with respect to $\rho_{0}$ at $\rho_{0}=0$ and not increasing along the process $\varphi$, i.e. $\dot{V} \leq 0$.

Theorem 6. [20] The process $\varphi$ with the equilibrium $\varphi_{0}=$ 0 is asymptotically stable with respect to the two discrepancies $\rho$ and $\rho_{0}$ if and only if there exists a functional $V=V[\varphi, t]$ positive definite with respect to the discrepancy $\rho$, continuous at time $t=t_{0}$ with respect to $\rho_{0}$ at $\rho_{0}=0$ and not increasing along the process $\varphi$, i.e. $\dot{V} \leq 0$, with $\lim _{t \rightarrow \infty} V=0$.

It has to be mentioned that stability with respect to two discrepancies is necessary but in general not sufficient for stability with respect to a $L_{p}$ norm or $L_{\infty}$ norm.

\section{ADAPTIVE DISCREPANCY BASED CONTROL DESIGN CONTROL DESIGN}

In the following a stabilizing control is derived for the fluidized bed spray granulation with internal classification (7). As has been shown earlier [8] the third moment of the particle size distribution $\mu_{3}$ as the controlled variable and the suspension injection rate $\dot{V}_{s}$ as the control variable are appropriate handles in order to stabilize the process. The error therefore is

$$
e=\int_{0}^{\infty} L^{3}\left(n_{d}-n\right) d L
$$

In order to derive a stabilizing controller the above presented stability concept is applied. Here, we choose the discrepancy $\rho$ as follows

$$
\rho=\frac{1}{2}\left(\int_{0}^{\infty} L^{3}\left(n_{d}-n\right) d L\right)^{2} .
$$

Obviously, the above requirements on a discrepancy are met. In order to guarantee continuity at time $t=t_{0}$ at $\rho_{0}=0$ the second discrepancy $\rho_{0}$ is simply chosen as follows

$$
\rho_{0}=\rho(t=0) \text {. }
$$

According to Theorem 6 existence of an appropriate functional $V$ is sufficient to guarantee asymptotic stability with respect to the two discrepancies $\rho$ and $\rho_{0}$. For this purpose the following candidate Lyapunov functional is introduced

$$
V=\frac{1}{2}\left(\int_{0}^{\infty} L^{3}\left(n_{d}-n\right) d L\right)^{2} .
$$

In order to account for the unknown parameter $k$ this candidate Lyapunov functional has to be augmented

$$
V=\frac{1}{2}\left(\int_{0}^{\infty} L^{3}\left(n_{d}-n\right) d L\right)^{2}+\frac{1}{2 \gamma} \tilde{k}^{2},
$$

where $\tilde{k}=\hat{k}-k$ is the estimation error. This approach, i.e. Lyapunov redesign, is well known for finite dimensional systems. In order to achieve stability in the sense described above the control variable has to be chosen such that the time derivative of $V$ along the system trajectories (7) is negative definite for all times and vanishs only for $V=0$.

Calculating the time derivative $\dot{V}$ yields

$$
\begin{aligned}
\dot{V}= & -e \int_{0}^{\infty} L^{3}\left(-G \frac{\partial n}{\partial L}-\dot{n}_{\text {prod }}+B\right) d L+\frac{1}{\gamma} \tilde{k} \dot{\hat{k}} \\
= & -e \int_{0}^{\infty} L^{3}\left(-\frac{2(1-b)}{\pi \mu_{2}} \frac{\partial n}{\partial L}+\frac{b}{\frac{1}{6} \pi} n_{B}(L)\right) k \dot{V}_{s} d L \\
& -e \int_{0}^{\infty} L^{3} K T(L) n d L+\frac{1}{\gamma} \tilde{k} \dot{\hat{k}} .
\end{aligned}
$$

In order to achieve negative definiteness of the time derivative of the candidate Lyapunov functional $V$ (15) the following certainty equivalence control law is chosen.

$$
\dot{V}_{s}=\frac{c e+\int_{0}^{\infty} L^{3} K T(L) n d L}{\hat{k} \int_{0}^{\infty} L^{3}\left(-\frac{2(1-b)}{\pi \mu_{2}} \frac{\partial n}{\partial L}+\frac{b}{\frac{1}{6} \pi} n_{B}(L)\right) d L}
$$

Applying the certainty equivalence control law in (15) results in

$$
\dot{V}=-c \frac{k}{\hat{k}} e^{2}-e \frac{\tilde{k}}{\hat{k}} \int_{0}^{\infty} L^{3} K T(L) n d L+\frac{1}{\gamma} \tilde{k} \dot{\hat{k}} .
$$

Here, the first term is clearly negative definite, as $k$ and $\hat{k}$ are always positive, and choosing an appropriate parameter adaption law, i.e. $\dot{\hat{k}}$, the remaining terms cancel. 


$$
\dot{\hat{k}}=\frac{\gamma}{\hat{k}} \int_{0}^{\infty} L^{3}\left(n_{d}-n\right) d L \int_{0}^{\infty} L^{3} K T(L) n d L
$$

In addition to stability with respected to the two discrepancies $\rho$ and $\rho_{0}$, the control law (16) guarantees exponential convergence of $V$.

$$
\dot{V}=-c \frac{k}{\hat{k}} e^{2}=-2 c \frac{k}{\hat{k}} V
$$

It has to mentioned that applying the discrepancy based control law (16) together with the proposed adaptation law (18) guarantees stability with respect to a $L_{p}$ or $L_{\infty}$ norm only if the zero dynamics associated with the discrepancy $\rho$ are stable with respect to a $L_{p}$ or $L_{\infty}$ norm, which is in accordance with $[17,18]$. As a rigorous stability analysis of the zero dynamics is difficult an heuristic approach is to study the zero dynamics of the linearized semi-discrete approximations. For the presented continuous fluidized bed spray granulation with internal product classification the zero dynamics are stable around the steady states studied in the one-parameter bifurcation analysis (Fig. 5). The control law as depicted in Fig. 6 consists of nonlinear compensation part, which needs a measurement of the particle size distribution $n$, a proportional error feedback and an adaptation law.

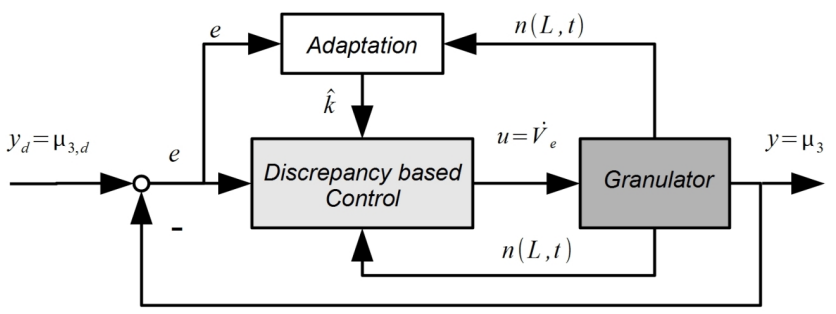

Fig. 6. Control scheme

In order to test the control law the effective suspension injection rate is varied by $\pm 20 \%$, i.e. $k=1 \pm 0.2$, in the unstable region, i.e. $\dot{V}_{e}=145000 \frac{\mathrm{mm}^{3}}{\mathrm{~s}}$. As can be seen in Fig. 7 and 8 the adaptive version of the discrepancy based control succeeds in stabilizing the desired particle size distribution with reasonable control effort (Fig. 9). This can also be observed in the bed height $h$ in Fig. 10. The adaptation of the unknown parameter $k$, i.e. the estimate $\hat{k}$, is shown Fig. 11 .

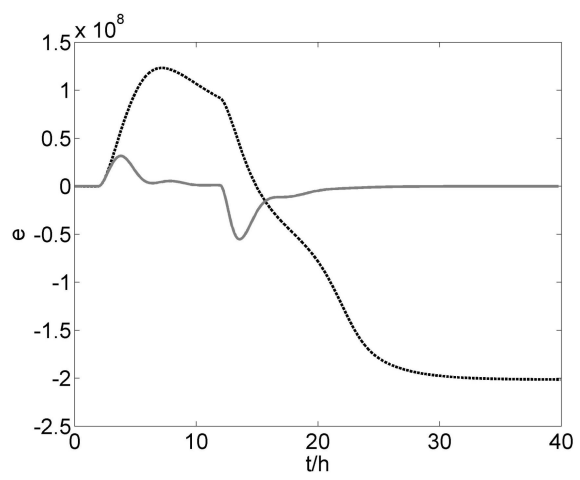

Fig. 7. Error in the particle size distribution $e$ with (gray) and without adaptation (black --)
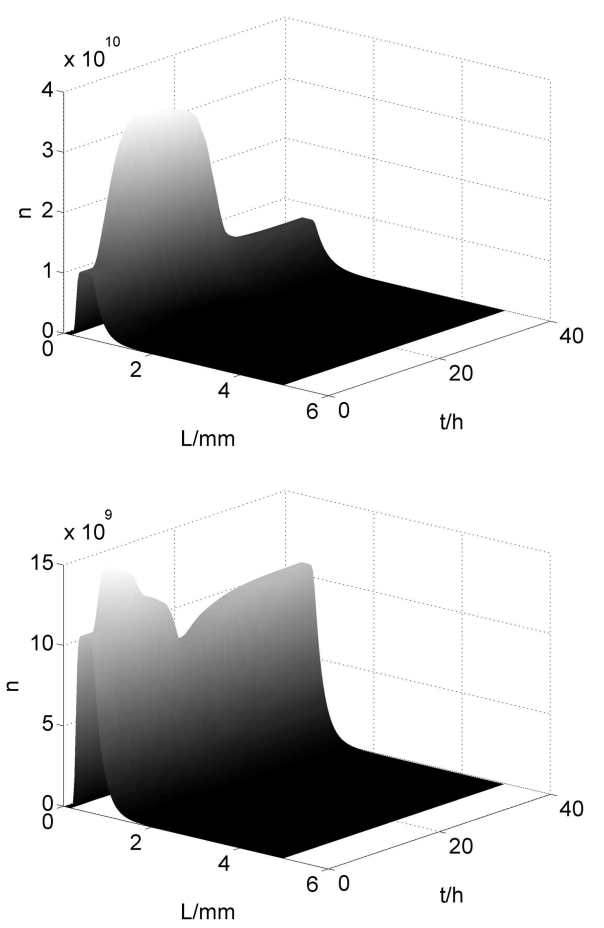

Fig. 8. Particle size distribution $n$ without (top) and with adaptation (bottom)

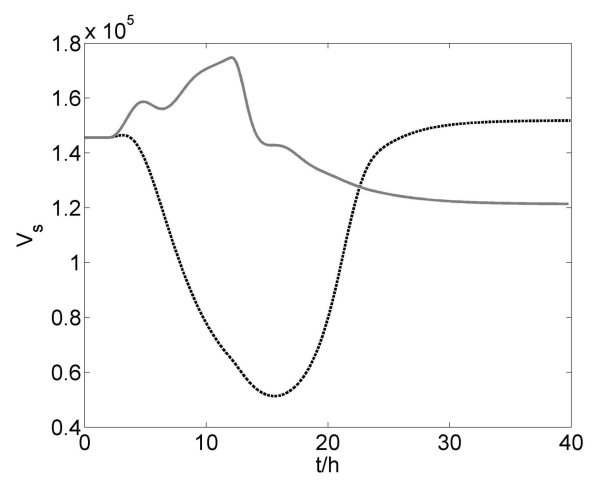

Fig. 9. Suspension injection rate $V_{s}$ with (gray) and without adaptation (black - - )

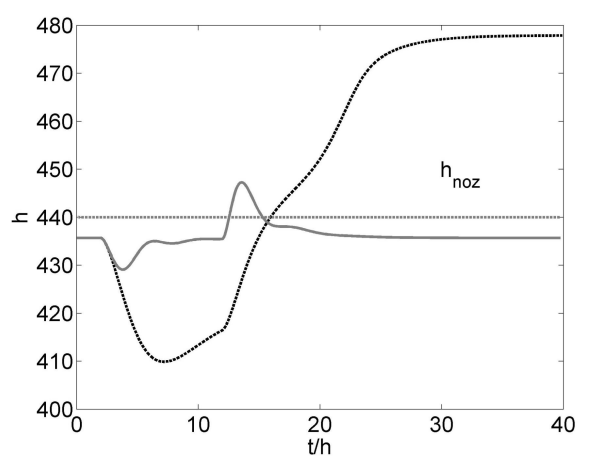

Fig. 10. Variation in the bed height $h$ with (gray) and without adaptation (black --) 


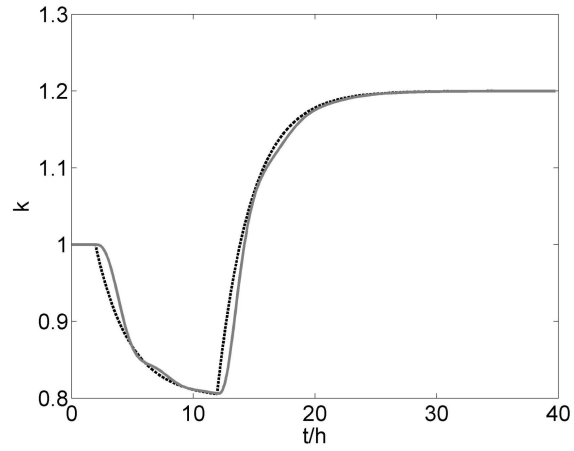

Fig. 11. Variation of $k$ (black--) and its adaptation $\hat{k}$ (gray)

\section{CONCLUSION}

For a continuous fluidized bed spray granulation with internal product classification and parametric uncertainty connected to varying drying conditions and variations in the liquid phase an adaptive nonlinear control approach has been proposed. The main idea is to augment the Lyapunov functional in order to account for the parameter estimate error $\tilde{k}$ and then derive a certainty equivalence control law using stability with respect to two discrepancies and an associate parameter adaptation law.

\section{REFERENCES}

[1] L. Mörl \& M. Mittelstrass \& J. Sachse, Zum Kugelwachstum bei der Wirbelschichttrocknung von Lösungen oder Suspensionen. Chem. Techn. 29, 1977, Heft 10, pp. 540-542.

[2] S. Heinrich \& M. Peglow \& M. Ihlow \& M. Henneberg \& L. Mörl, Analysis of the start-up process in continuous fluidized bed spray granulation by population balance modeling, Chem. Eng. Sci. 57, 2002, pp. 4369-4390.

[3] R. Radichkov \& T. Müller \& A. Kienle \& S. Heinrich \& M. Peglow \& L. Mörl, A numerical bifurcation analysis of continuous fluidized bed spray granulation with external product classification, Chem. Eng. Proc., Proc. 45, 2006, pp. 826-837.

[4] A.W. Vreman \& C.E. van Lare \& M.J. Hounslow, A basic population balance model for fluid bed spray granulation, Chem. Eng. Sci. 64, 2009, pp. 4389-4398.

[5] S. Palis \& A. Kienle, Stabilization of continuous fluidized bed spray granulation - a Lyapunov approach, 8th IFAC Symposium on Nonlinear Control Systems - NOLCOS, 2010, 1362-1367.

[6] S. Palis \& A. Kienle, Diskrepanzbasierte Regelung der kontinuierlichen Kristallisation, at-Automatisierungstechnik, 2012, pp. $145-154$

[7] S. Palis \& A. Kienle, Stabilization of continuous fluidized bed spray granulation with external product classification, Chem Eng. Sci., 2012, pp. 200-209.

[8] S.Palis \& A. Kienle, $H_{\infty}$ loop shaping control for continuous fluidized bed spray granulation with internal product classification, Industrial \& Engineering Chemistry Research, 2013, pp. 408-420.

[9] S. Palis \& A. Kienle, Discrepancy based control of continuous fluidized bed spray granulation with internal product classification, 8th IFAC International Symposium on Advanced Control of Chemical Processes - ADCHEM, 2012, pp. 756-761.

[10] R. Eek, Control and dynamic modeling of industrial suspension crystallizers, PhD thesis, TU Delft, 1995.

[11] P. Christofides \& N. El-Farra \& M. Li \& P. Mhaskar, Modelbased control of particulate processes, Chem. Eng. Sci. 63, 2008, pp. 1156-1172.
[12] U. Vollmer \& J. Raisch, Population balance modeling and $H_{\infty}$ controller design for a crystallization process, Chem. Eng. Sci., vol. 57, issue 20, Oct. 2002, pp. 4401-4414.

[13] A. D. Randolph \& M. A. Larson, Theory of particulate processes, New York: Academic Press, Inc., 1988.

[14] A. Smyshlyaev \& E. Cerpa \& M. Krstic, Boundary stabilization of a 1-D wave equation with in-domain anti-damping, SIAM Journal of Control and Optimization, vol. 48, pp. 4014-4031, 2010.

[15] J-M. Coron \& B. d'Andra-Novel \& G. Bastin, A strict Lyapunov function for boundary control of hyperbolic systems of conservation laws, IEEE Transactions on Automatic Control, Vol. 52(1), pp. 2 - 11, 2007.

[16] C. Prieur \& J. Winkin \& G. Bastin, Robust boundary control of systems of conservation laws, Mathematics of Control, Signals and Systems, Vol. 20, pp. 173 - 197, 2008.

[17] C. I. Byrnes, D. S. Gilliam, Boundary feedback design for nonlinear distributed parameter systems, Conference on Decision and Control - CDC, 1991.

[18] C. I. Byrnes, D. S. Gilliam, V. I. Shubov, Boundary control, stabilization and zero-pole dynamics for a non-linear distributed parameter system, Int. J. RobustNonlinear Control, 1999, pp. 737-768.

[19] A.A. Movchan, Stability of processes with respect to two metrics, Journal of Applied Mathematics and Mechanics, 1960, 24, pp. 1506-1524.

[20] T. Sirasetdinov, Stability of systems with distributed parameters, 1987.

[21] A. Martynjuk \& R. Gutovski, Integral inequalities and stability of motion, Naukowa Dumka, 1979. 